\title{
El médico residente como educador
}

\section{The resident as an educator}

\author{
Guinchad y Sánchez E* \\ Colegio Mexicano de Ortopedia y Traumatología.
}

RESUMEN. En la enseñanza de la medicina, los médicos residentes desempeñan un papel muy importante, ya que en muchas ocasiones son quienes tienen que dar las clases a los estudiantes de medicina, internos rotatorios de pregrado y a los residentes de menor jerarquía. En ocasiones, su preparación como docentes no es la adecuada, por lo que es muy importante la inclusión de este tema en su currículum académico, con el fin de profesionalizar al docente. La División de Postgrado de la Facultad de Medicina de la Universidad Nacional de México incluyó en el Programa Único de Especializaciones Médicas (PUEM) desde 1994, el Seminario de Educación Médica y desde hace dos años este Seminario se lleva en línea, tiene carácter obligatorio tanto para los médicos residentes como los profesores. Sin embargo, es necesario dar mayor importancia a la formación de los médicos residentes como docentes, esta acción llevará a una mejor preparación de los estudiantes de pregrado, internos rotatorios, los residentes mismos y estimulará a los profesores a mejorar y profesionalizar su carácter de docentes.

Palabras clave: Enseñanza, médicos residentes, profesionalización docente.

Haz lo mismo que hacen los demás, pero poniendo toda tu alma en ello porque el resultado será totalmente diferente.

Miguel de Unamuno

(1864 Bilbao-1936 Salamanca)
ABSTRACT. In teaching medicine, resident physicians play a very important role, as on many occasions they are the ones who have to teach the medical students, undergraduate rotary interns and residents of other years. Sometimes his preparation as teachers is not adequate, so it is very important to include this topic in his academic curriculum, in order to professionalize the teacher. The Graduate Division of the School of Medicine of the National Autonomous University of Mexico (UNAM in spanish) included in the Single Program of Medical Specializations (PUEM in spanish) since 1994, the Seminar of Medical Education and for two years this seminar is carried online, has mandatory character for both resident doctors and teachers. However, it is necessary to give greater importance to the training of resident physicians as teachers, this action will lead to better preparation of undergraduate students, rotary interns, residents themselves and will encourage teachers to improve and professionalize their teaching character.

Keywords: Teaching, medical residents, professional learning in medicine.

A través de los años de experiencia hospitalaria, primero como estudiante de pregrado, luego como interno rotatorio de pregrado, como médico residente en preparación de una especialización y posteriormente como médico de base y aun como jefe de enseñanza de un importante hospi\footnotetext{
el Área de la Salud del Colegio Mexicano de Ortopedia y Traumatología, A.C.

Correspondencia:

Dr. Enrique Guinchad y Sánchez

Durango 33, Tercer piso, Consultorio 30-A, Col. Roma Norte, C.P. 06700,

Alcaldía Cuauhtémoc, Ciudad de México.

E-mail: r_sanfrancisco@yahoo.com.mx
}

* Miembro Emérito del Colegio Mexicano de Ortopedia y Traumatología. Profesor adjunto del Diplomado de Formación de Profesores en 
tal escuela, nos hemos percatado de que uno de los pilares fundamentales de la enseñanza está en manos de los médicos residentes, a quienes encargamos o delegamos nuestras funciones para que eduquen a los individuos de este proceso en los diferentes escalafones de la enseñanza de pregrado y postgrado.

Tan es así que varias universidades y escuelas de medicina han valorado la necesidad de una adecuada formación como docente del médico residente. El ejemplo que conozco es el de la División de Estudios Superiores de la Facultad de Medicina de la Universidad Nacional Autónoma de México, que inició el Programa Único de Especialidades Médicas y que desde 1994 incluyó el Seminario de Educación Médica, el cual en la actualidad se imparte en línea y cuenta con muy buena planeación. Su propósito es un mayor desarrollo de los residentes en el tema y también es obligatorio para los profesores titulares y adjuntos (considerado como un requisito para los nombramientos de profesores), cubriendo así otra muy importante necesidad: la de profesionalizar a los profesores en el área de la docencia. 1,2,3,4,5

Precisamente por este motivo es fundamental que se trate el tema de la relevancia del médico residente como educador, debiendo ser esta característica una faceta esencial de la formación de un buen médico en toda especialidad de cualquiera de las disciplinas que se manejan en la formación de postgrado.

Estudios realizados tanto en nuestro país como en el extranjero coinciden en que los mismos médicos residentes actúan como responsables de la enseñanza de otros individuos en formación y especialmente de sus compañeros de años inferiores en una proporción significativa. En nuestro país, en algunos sitios esta proporción es aún más importante, lo que hace surgir una interrogante: ¿está realmente capacitado el médico residente para llevar a cabo su función docente?

Si la respuesta es sí, podemos estar tranquilos con respecto a su actividad; sin embargo, ésta siempre debe ser guiada por los profesores, los cuales tienen que estar debidamente capacitados como docentes. ${ }^{6,7}$

En caso contrario, estamos haciendo culpa nuestra que la mala preparación se reproduzca en forma exponencial y esto lleva a una mala preparación de estudiantes de pregrado y postgrado, sabiendo que es causa de una mala preparación de los profesores por falta de interés hacia la docencia, no así a los nombramientos de gran reconocimiento social. Es aquí donde se debe insistir en la preparación adecuada del docente como tal.

El médico residente por definición es el estudiante de posgrado, cuya función es aprender de sus docentes (maestros), que se supone son los que mayor experiencia tienen en el campo médico y como docentes en la transmisión del conocimiento y de sus complejas destrezas ${ }^{6}$ que podemos definir como «la praxis: teoría, práctica y regreso a la teoría», que es un fundamento importante en el desarrollo cognitivo, sicomotriz e incluso afectivo, al que podemos aplicar el principio de Ausebell de «aprendizaje significativo», es decir, el que ocasiona un cambio significativo en el sujeto de la enseñanza (el alumno).

Pero surge una duda: ¿realmente esto sucede así? y la respuesta es que en la formación nuestros compañeros de mayor jerarquía eran quienes en la mayoría de las veces actuaban como profesores, con ellos vivimos en los hospitales, en sus salas de internamiento, en sus quirófanos y en sus consultas externas y en muchos casos fueron de ellos de quienes aprendimos nuestras primeras destrezas y conocimientos siempre bajo su supervisión. ${ }^{5}$

Esto es sumamente importante, ya que si bien o mal aprendimos, pero ahora reconocemos que se hizo ese proceso docente sin conocimientos básicos de la enseñanza y podemos deducir que si ésta hubiera existido, el proceso de aprendizaje hubiera sido mucho más satisfactorio tanto para el que enseña como para el que aprende.

Los médicos residentes tienen como una de sus funciones inherentes a su cargo el proceso de enseñanza hacia los estudiantes de pregrado y sus compañeros de residencia, que es agradable para ellos y la aceptan como una manera de ir adquiriendo nuevos concomimientos.

En un estudio realizado en 2005 por la División de Estudios Superiores de la Facultad de Medicina de la Universidad Nacional Autónoma de México, en el cual se aplicó una encuesta al respecto de la participación de los médicos residentes como docentes aplicado en sus 75 especializaciones se obtuvo un total de 5,176 encuestas, en las que se preguntó: 1 . La percepción que tenían sobre la capacidad para educar estudiantes, internos y a otros residentes. 2. El grado de conocimientos que consideraban haber recibido de otros residentes. 3. La percepción que tenían de su importancia como educadores. 4. Sobre la necesidad de recibir instrucción dirigida para mejorar sus capacidades docentes. ${ }^{8}$

Los resultados resumidos fueron:

1. $43 \%$ aceptaron que más de $2 / 3$ de sus conocimientos y destrezas provenían de las enseñanzas de residentes de años superiores.

2. De los 4,833 estudiantes de medicina, 4,561 estuvieron de acuerdo en que la mayor parte de conocimientos y destrezas les fueron enseñadas por los médicos residentes. Sólo 212 no contestaron o estuvieron en desacuerdo.

3. De los 4,819 internos rotatorios de pregrado 4,644 estuvieron de acuerdo en que la mayor parte de sus conocimientos $\mathrm{y}$ destrezas les fueron enseñadas por médicos residentes y sólo 175 no estuvieron de acuerdo o lo desconocían.

4. De los 4,854 residentes, 3,512 estuvieron en total acuerdo, 1,158 de acuerdo, 78 lo desconocían, 84 en desacuerdo y 13 en total desacuerdo. Como podemos ver la mayoría $(4,670)$ estuvieron de acuerdo en que la mayor parte de sus conocimientos y destrezas les fueron enseñadas por médicos residentes.

5. Percepción de los médicos residentes con respecto a la necesidad de recibir instrucción sobre estrategias de enseñanza en el área de la salud, de 4,840 encuestas se obtuvo: 2,898 completo acuerdo, 1,544 de acuerdo, no lo sabían 
241 , en desacuerdo 114 y en total desacuerdo 43. En total 4,442 estuvieron de acuerdo en recibir preparación docente.

6. Con relación a la facilidad de llevar a efecto los cursos de formación docente, los principales obstáculos fueron falta de tiempo y exceso de trabajo.

7. De las preferencias en metodología didáctica: $44 \%$ conferencias, $28 \%$ enseñanza interactiva en grupos reducidos, $14 \%$ cursos en línea, $8 \%$ lecturas programadas, sesiones bibliográficas, $5 \%$ cursos interactivos y grupos interactivos (como podemos ver los conocimientos de métodos y técnicas de la enseñanza no son muy integrados).

8. Con respecto al tiempo que pudieran emplear, se observó que $29 \%$ sugiere dos horas, $23 \%$ proponen cuatro horas, $20 \%$ seis horas, $8 \%$ ocho horas, $11 \% 10$ horas y $8 \% 24$ horas a la semana. ${ }^{9}$

Con estos resultados obtenidos por la Facultad de Medicina de la Universidad Nacional Autónoma de México podemos darnos cuenta de la relevancia del médico residente como educador; sin embargo, nos encontramos con el problema del tiempo que se puede dedicar a la formación del médico residente como profesor, por lo que se sugiere que en los centros formadores de recursos humanos para la salud se dé oportunidad para que además de llevar los seminarios sobre educación que se toman en línea, se les permita asistir a cursos cuya meta es la formación de docentes en el área de la salud.

Sin embargo, no hay un programa universal para la profesionalización del residente como docente. En los países del norte del Continente Americano tenemos que en el Royal College of Physicians of Canada, en el Accreditation Council for Graduate Medical Education (ACGMA) de los Estados Unidos y en el Programa Único de Especializaciones Médicas de la Universidad Nacional Autónoma de México señalan la necesidad de establecer competencias educativas para los especialistas; sin embargo, no está claro cómo deben ser estas competencias en educación y menos aún cómo las deben desarrollar las universidades, existiendo tan sólo 55\% de las residencias en Estados Unidos que contemplan esta necesidad imperiosa de capacitar a los médicos residentes en docencia, ${ }^{6,7}$ mientras que en los programas de la Universidad Nacional Autónoma de México actualmente se cubre a $100 \%$ de los inscritos en cursos de especialización.

En general los programas existentes en las diferentes facultades o escuelas de medicina no guardan ninguna relación entre sí, lo cual es explicable por la idiosincrasia de las diferentes regiones que se incluyen en el estudio mencionado.

En la actualidad en algunos lugares se consideran los contenidos temáticos, los enunciados por Bringing Education and Service Together (BEST) de la Universidad de California en Irvine, ${ }^{10,11}$ que tiene varios años de experiencia y que propone algunos contenidos didácticos que deben cubrirse por semestre con 13 horas de trabajo:
1. Liderazgo y papel modulador de conductas.

2. Cómo orientar a los alumnos (aprendices).

3. Cómo dar retroalimentación positiva.

4. La enseñanza en la cabecera del paciente.

5. Procedimientos de enseñanza-aprendizaje.

6. Enseñanza en las salas de internamiento.

7. Enseñanza a través del expediente clínico (casos clínicos, investigación).

8. Enseñar a dar conferencias y clases (residente-profesor).

Por tal motivo, la enseñanza debe quedar como parte real del currículo de adiestramiento del médico residente y no como parte de ese currículo oculto (también tan importante), aunque en la actualidad los contenidos clínicos son tan extensos que poco tiempo dejan para incluir estos temas sobre las técnicas de la enseñanza y aprendizaje, lo cual nos lleva a pensar en elaborar una adecuada programación entre los temas clínicos, quirúrgicos y relativos a la ética, educación e investigación ${ }^{12}$ con el fin de formar médicos con un enfoque integral, promoviendo la vinculación entre la teoría y la práctica (praxis), la adquisición de aprendizajes significativos en la medicina, la docencia y la investigación, todo con compromiso social y ético, siendo ésta una tarea ya impostergable. ${ }^{13,14}$

Puede haber otros puntos a tratar, por lo que se deben definir adecuadamente los temas primordiales para la actividad docente del médico residente mediante cursos en línea, semipresenciales o presenciales, utilizando discusiones coordinadas, talleres, mesas redondas, etcétera. Y se debe evaluar constantemente el efecto de los cursos, con el fin de detectar las necesidades reales y emergentes de cada grupo para permitir un adecuado desempeño de los médicos residentes como educadores en el proceso de formación de recursos humanos en el área de la salud.

\section{Conclusiones}

De acuerdo a la experiencia nacional y mundial, el médico residente desempeña un papel importante en la enseñanza de la medicina, tanto con estudiantes, internos rotatorios de pregrado y demás médicos residentes en mayor o menor tiempo y con mayores o menores aptitudes, lo que redunda en el proceso de enseñanza-aprendizaje de la medicina tanto general como de especializaciones.

La División de Estudios Superiores de la Facultad de Medicina de la Universidad Nacional Autónoma de México así como otras instituciones formadoras de recursos humanos para la salud han detectado el problema y le han dado solución elaborando un curso en línea del Programa Único de Especialidades Médicas a través del Seminario de Educación. Sin embargo, es necesario darle al médico residente la oportunidad de tomar cursos de formación de profesores, buscando una mejor participación en la docencia y profesionalizándola de una manera activa y constante. Es esencial que los médicos residentes participen en los cursos de formación de profesores en el área de la salud. 
Los programas de formación de docentes deben ser dinámicos, ajustarse a las necesidades observadas en la retroalimentación de cada programa y ser idóneos para cada institución o región de acuerdo con sus necesidades en la formación de docentes entre los médicos residentes.

La mejor capacitación del médico residente como docente nos beneficiará a todos al tener médicos más capacitados y con gran enfoque humanista, adiestrándolos para la transmisión del conocimiento en una forma profesional y ética.

Referencias

1. Plan Único de Especialidades Médicas (PUEM), División de Estudios Superiores. Facultad de Medicina. Universidad Nacional Autónoma de México. Ortopedia.

2. Rivero SO. Educación médica: concepto y motivación. En: Graue WE, Sánchez MM, Durante MI, Rivero SO. Educación en las residencias médicas. Facultad de Medicina. Universidad Nacional Autónoma de México. Editores de Textos Mexicanos, S.A. de C.V. México. 2010, 3-8.

3. Méndez-López JF, Mendoza-Espinosa H, Torruco-García T, SánchezMendiola M. El médico residente como educador. Inv Ed Med. 2013; 2(7): 154-61.

4. Aréchiga UH, Ruiz PL, Durand OP, Sánchez RR, Gracia RA. El plan único de especializaciones médicas de la facultad de medicina. Rev Fac Med UNAM. 2000; 43(1): 19-23.

5. Sánchez-Mendiola M. El Seminario de Educación del Plan Único de Especializaciones Médicas de la UNAM, una reflexión crítica. Los Retos de la Educación Médica. 2012; 1(1): 135-62.

6. Guinchard SE, Cansino QI Diplomado de formación de profesores en el área de la salud. Acta Ortop Mex. 2016; 30(5): 221-2.
7. Ponce LM. Formación de profesores. En: Pérez CVJ, Lavalle M. La educación médica en el siglo XXI. Academia Mexicana de Cirugía. Colección Platino. Editorial Alfil. México. 2009, 13-24.

8. Graue WE, Ponce LM, Sánchez MM. El residente como educador médico. En: Graue WE, Sánchez MM, Durante MI, Rivero SO. Educación en las residencias médicas. Facultad de Medicina. Universidad Nacional Autónoma de México. Editores de Textos Mexicanos, S.A. de C.V. México. 2010. 79-91.

9. Morrison EH, Friedland JA, Bocker J, Rucker L, Hollingshead J. Murata P. Residents as teachers training in US residency programs and offices of graduate medical education. Acad Med. 2001; 76: 51-4.

10. Graue WE, Sánchez MM. El papel docente en la medicina de los residentes. En: Pérez CJ, Lavalle MC. La educación médica en el siglo $X X I$. Academia Mexicana de Cirugía. Colección Platino. Editorial Alfil. México. 2009, 103-112.

11. Morrison EH, Rucker LJ, Boker JR, Gabbert CC, Hubbell FA. The effect of a 13-hour curriculum to improve resident's teaching skills. Ann Intern Med. 2004; 141(4): 257-63.

12. Kershenobich SD. El reto educativo de ser residente. En: Graue WE, Sánchez MM, Durante MI, Rivero SO. Educación en las residencias médicas. Facultad de Medicina. Universidad Nacional Autónoma de México. Editores de Textos Mexicanos, S.A. de C.V. México. 2010, 93-99.

13. Moreno-Altamirano L, García-García J, Urbina-Cedillo C, Garcíade la Torre GS. Actividad docente facilitadora para la adquisición de aprendizajes significativos y compromiso social. Inv Ed Med. 2013; 2(7): $142-7$.

14. Heinz-Martín G, Olmedo-Canchola VH, Bazán-Miranda G, Guinchad y SE, Torres-Carrillo J. Aspectos relevantes de los cursos de especialización en Ortopedia avalada por la Universidad Nacional Autónoma de México. Acta Ortop Mex. 2019; 33(3): 192-6.

Conflicto de intereses: Ninguno. 